\title{
ON THE EXISTENCE OF AUTOMORPHIC QUASIMEROMORPHIC MAPPINGS IN $R^{n}$
}

\author{
O. MARTIO and U. SREBRO
}

\section{Introduction}

Let $G$ be a Möbius group in $\bar{R}^{n}=R^{n} \cup\{\infty\}, n \geqq 2$, and $D$ a domain in $R^{n}$. A mapping $f: D \rightarrow \bar{R}^{n}$ is said to be automorphic with respect to $G$ if $f$ is continuous, open, discrete, sense-preserving, and $f \circ \mathrm{g}=f$ for all $g \in G$. Note that if $G$ has automorphic mappings $f: D \rightarrow \bar{R}^{n}$, then $G$ is discrete and $D$ is invariant under $G$. Discrete Möbius groups which have invariant domains are called function groups.

A mapping $f: D \rightarrow R^{n}$ is called quasiregular, abbreviated $q r$, if $f$ is continuous, $\mathrm{ACL}^{n}$, and

$$
\left|f^{\prime}(x)\right|^{n} \leqq K J(x, f)
$$

a.e. in $D$ for some $K \in[1, \infty)$. If $f: D \rightarrow \bar{R}^{n}$, then the $\mathrm{ACL}^{n}$ condition and (1.1) can be checked at $f^{-1}(\infty)$ by means of auxiliary Möbius transformations. If these conditions hold the mapping is then said to be quasimeromorphic, abbreviated qm. If $n=2$ and (1.1) holds with $K=1$, then $f$ is meromorphic.

The purpose of this note is to prove

1.2. Theorem. Let $G$ be a discrete Möbius group acting on $B^{n}, n \geqq 2$, with $V\left(B^{n} / G\right)<\infty$. Then $G$ has qm automorphic mappings $f: B^{n} \rightarrow \bar{R}^{n}$.

In the above theorem $V\left(B^{n} / G\right)$ denotes the hyperbolic volume of the orbit space $B^{n} / G$, see [4].

The proof is constructive. It is based on a modification of the method of Alexander [1], on basic properties of Möbius groups, see Chapter 3, and on the properties of radial strecthings, see Chapter 2. We shall not estimate the dilatations of $f$ in terms of $G$. For the sake of simplicity we shall restrict ourselves to the case $n=3$. The same method applies to $n>3$ and to $n=2$.

It is known that every function group in $R^{2}$ has meromorphic automorphic mappings. We do not know whether function groups in $R^{n}, n>2$, have qm automorphic mappings, nor we know whether the condition $V\left(B^{n} / G\right)<\infty$ in Theorem 1.2 is essential. We have examples, see $[4,4.2]$, of $q m$ automorphic mappings $f: B^{n} \rightarrow \bar{R}^{n}$ for infinite groups with $V\left(B^{n} / G\right)=\infty$.

doi:10.5186/aasfm.1977.0317 
The notation and terminology will be as in [4]. In particular we denote $x=\left(x_{1}, \ldots, x_{n}\right)=\sum x_{i} e_{i}$ for $x \in R^{n}, B^{n}(a, r)=\left\{x \in R^{n}:|x-a|<r\right\}, B^{n}(r)=B^{n}(0, r)$, $B^{n}=B^{n}(1), \quad S^{n-1}(a, r)=\partial B^{n}(a, r), \quad S^{n-1}(r)=S^{n-1}(0, r), \quad S^{n-1}=S^{n-1}(1), \quad H^{n}(h)=$ $=\left\{x \in R^{n}: x_{n}>h\right\}$, and $H^{n}=H^{n}(0)$. For Möbius groups $G$ acting on $B^{n}$ we let Fix $G=\left\{x \in B^{n}: g(x)=x\right.$ for some $\left.g \in G \backslash\{\mathrm{id}\}\right\}$.

\section{Radial stretchings}

2.1. In this chapter we consider a special class of bi-lipschitzian mappings. A mapping $f: A \rightarrow R^{n}, A \subset R^{n}$, is called bi-lipschitzian if

$$
|x-y| / L \leqq|f(x)-f(y)| \leqq L|x-y|
$$

for all $x$ and $y$ in $A$ and for some $L \geqq 1$. The smallest $L$ for which (2.2) holds will be denoted by $L(f)$.

2.3. A bounded domain $D \subset R^{n}$ is said to be strictly star shaped if each ray $L$ from 0 meets $\partial D$ at exactly one point. It follows that $0 \in D$ and that the mapping $\varphi^{*}: \partial D \rightarrow S^{n-1}$ which sends $L \cap \partial D$ to $L \cap S^{n-1}$ is a homeomorphism. We let $\varphi: R^{n} \rightarrow R^{n}$ denote the radial linear extension of $\varphi^{*}$, i.e. $\varphi(x)=x \varphi^{*}\left(x^{*}\right) /\left|x^{*}\right|, x \neq 0$, and $\varphi(0)=0$ where $\left\{x^{*}\right\}=\partial D \cap\{t x: t>0\}$. This mapping $\varphi$ which is an automorphism of $R^{n}$ and maps $D$ onto $B^{n}$ will be called the radial linear stretching defined by $D$.

2.4. Lemma. Suppose that $D$ is strictly star shaped and $\varphi^{*}: \partial D \rightarrow S^{n-1}$ is bi-lipschitzian. Then $\varphi$ is bi-lipschitzian.

Proof. Let $M=\sup \{|x|: x \in \partial D\}$ and $m=\inf \{|x|: x \in \partial D\}$. Let $x, y \in R^{n}$. We may assume that $x \neq 0$ and that $\varphi(x)=x$ i.e. $\varphi^{*}\left(x^{*}\right)=x^{*}$ since otherwise we consider the mapping $\varphi \circ F_{x}$ where $F_{x}(z)=\left|x^{*}\right| z$. Then $F_{x}$ is bi-lipschitzian with $L\left(F_{x}\right) \leqq \max (M, 1 / m), \varphi \circ F_{x}(x)=x$, and $\varphi$ is bi-lipschitzian if and only if $\varphi \circ F_{x}$ is. Let $\alpha \in[0, \pi]$ denote the angle between the vectors $x$ and $y$. If $y=0$ we set $\alpha=0$. We claim that

$$
|\varphi(y)-y| \leqq|y|\left(L\left(\varphi^{*}\right)+1\right) \alpha / m .
$$

If $y=0$ then $(2.5)$ is trivial. Suppose $y \neq 0$. Now

$$
\begin{aligned}
|\varphi(y)-y| & =|y|\left|\varphi^{*}\left(y^{*}\right)-y^{*}\right| /\left|y^{*}\right| \\
& \leqq|y|\left(\left|\varphi^{*}\left(y^{*}\right)-x^{*}\right|+\left|y^{*}-x^{*}\right|\right) / m \\
& \leqq|y|\left(\left|\varphi^{*}\left(y^{*}\right)-\varphi^{*}\left(x^{*}\right)\right|+L\left(\varphi^{*}\right)\left|\varphi^{*}\left(y^{*}\right)-\varphi^{*}\left(x^{*}\right)\right|\right) / m \\
& \leqq|y|\left(\alpha+L\left(\varphi^{*}\right) \alpha\right) / m
\end{aligned}
$$

and (2.5) follows. 
To prove that $|\varphi(x)-\varphi(y)| \leqq K|x-y|$ suppose first that $\alpha \geqq \pi / 2$. Then $|x-y| \geqq$ $|y|$ and (2.5) yields

$$
|\varphi(x)-\varphi(y)| /|x-y| \leqq 1+\pi\left(L\left(\varphi^{*}\right)+1\right) / m .
$$

If $\alpha<\pi / 2$, then $|x-y| \geqq|y| \sin \alpha$ and (2.5) implies

$$
\begin{aligned}
|\varphi(x)-\varphi(y)| /|x-y| & \leqq 1+\alpha\left(L\left(\varphi^{*}\right)+1\right) /(m \sin \alpha) \\
& <1+\pi\left(L\left(\varphi^{*}\right)+1\right) / m .
\end{aligned}
$$

To prove the opposite inequality let $x, y \in R^{n} \backslash\{0\}$. Then

$$
|\varphi(x)-\varphi(y)|=|| x\left|\varphi^{*}\left(x^{*}\right) /\right| x^{*}|-| y\left|\varphi^{*}\left(y^{*}\right) /\right| y^{*}|| \geqq|x-y| / M
$$

and since this inequality is trivial when $x=0$ or $y=0$, the lemma follows.

2.6. Let $D$ be a bounded domain in $R^{n}$ with $0 \in D$ and let $\beta \in(0, \pi / 4]$. We say that $D$ satisfies the $\beta$-cone condition if the open cone

$$
C(x, \beta)=\left\{z \in R^{n}:|z-x|<|x|,(x-z) \cdot x>|x-z||x| \cos \beta\right\}
$$

with vertex $x$ and central angle $\beta$ lies in $D$ whenever $x \in \partial D$. Note that if $D$ satisfies the $\beta$-cone condition, then $D$ is strictly star shaped.

2.7. Lemma. Suppose that $D$ satisfies the $\beta$-cone condition for some $\beta>0$. Then $\varphi^{*}$ is bi-lipschitzian.

Proof. Let $x, y \in \partial D$. Then

$$
\left|\varphi^{*}(x)-\varphi^{*}(y)\right| \leqq|x-y| / m
$$

where $m=\inf \{|z|: z \in \partial D\}$.

To prove the other inequality we may assume that $\varphi^{*}(x)=x$ and $|y| \leqq|x|$. Let $\alpha \in(0, \pi]$ denote the angle between $x$ and $y$. Suppose first that $\alpha \geqq \pi / 2$. Then

$$
\left|\varphi^{*}(x)-\varphi^{*}(y)\right| \geqq \sqrt{2} \geqq|x-y| / \sqrt{2} .
$$

Suppose now that $\alpha<\pi / 2$. Since $y$ is outside the cone $C(x, \beta)$, elementary trigonometry yields

$$
|x-y| \leqq|y| \sin \alpha / \sin \beta \leqq\left|\varphi^{*}(x)-\varphi^{*}(y)\right| / \sin \beta,
$$

and the lemma follows.

Since every bi-lipschitzian mapping of $R^{n}$ is quasiconformal, see [3], the above lemmas imply:

2.8. Corollary. Suppose that $D$ satisfies the $\beta$-cone condition for some $\beta>0$. Then the radial linear stretching $\varphi: R^{n} \rightarrow R^{n}$ defined by $D$ is quasiconformal.

2.9. Remarks. (a) We shall mainly use Corollary 2.8 to show that the homeomorphism $\varphi \mid D$ onto $B^{n}$ is quasiconformal. 
(b) It is easy to see that if $D$ is strictly star shaped, then $\varphi$ is bi-lipschitzian if and only if $D$ satisfies the $\beta$-cone condition for some $\beta>0$. Moreover, for $n=3$ the cone condition is equivalent to the boundary condition of $[3,5.3]$.

(c) We shall later use the following elementary property of linear stretchings: Let $D_{1}$ and $D_{2}$ be strictly star shaped domains and $\varphi_{1}, \varphi_{2}$ the corresponding linear stretchings. Define $\varphi=\varphi_{2}^{-1} \circ \varphi_{1}$. Suppose that $x, y \in \partial D_{1}$ and $E(x)=y$ for some $E \in O(n)$. If $|\varphi(x)|=|\varphi(y)|$, then $E \circ \varphi(x)=\varphi \circ E(x)$.

\section{Fundamental polyhedra for Möbius groups}

3.1. Normal fundamental polyhedra. Let $G$ be a discrete Möbius group acting on $B^{n}$. Then $G$ is countable and thus $B^{n} \backslash$ Fix $G \neq \emptyset$. The normal fundamental polyhedron $P$ centered at a point $x_{0} \in B^{n} \backslash$ Fix $G$ is defined by

$$
P=\left\{x \in B^{n}: d\left(x, x_{0}\right)<d\left(x, g\left(x_{0}\right)\right) \text { for all } g \in G \backslash\{\text { id }\}\right\} .
$$

Here $d$ denotes the hyperbolic distance in $B^{n} . P$ is a convex polyhedron in the hyperbolic sense, possibly with infinite number of faces. Each $(n-1)$-face, considering only $\partial P \cap B^{n}$, lies in a hyperbolic $(n-1)$-plane

$$
H\left(A, x_{0}\right)=\left\{x \in B^{n}: d\left(x, x_{0}\right)=d\left(x, A\left(x_{0}\right)\right)\right\}
$$

for some $A \in G \backslash\{$ id $\}$. Since $A H\left(A^{-1}, x_{0}\right)=H\left(A, x_{0}\right)$, the $(n-1)$-faces of $P$ are pairwise $G$-equivalent. Note also that $H\left(A^{-1}, 0\right)$ is contained in the isometric sphere $I(A)=\left\{x \in R^{n}:\left|A^{\prime}(x)\right|=1\right\}$ of $A$ and $A=E \circ I$ where $I$ is the reflection in $H\left(A^{-1}, 0\right)$ and $E$ is an orthogonal transformation in $R^{n}$. Indeed, $\left(I \circ A^{-1}\right) B^{n}=B^{n}$ and $\left(I \circ A^{-1}\right)(0)=0$ and so $I \circ A^{-1} \in O(n)$. Therefore $A=E \circ I$ for some $E \in O(n)$, and $\left|A^{\prime}(x)\right|=1$ for all $x \in H\left(A^{-1}, 0\right)$. Finally, recall that if $G$ is discrete and the hyperbolic measure $V\left(B^{n} / G\right)$ is finite, then, see [2], [5], or [6], every normal fundamental polyhedron $P$ has finitely many faces and $\bar{P} \cap S^{n-1}$ is either empty, which happens only when $B^{n} / G$ is compact, or consists of finitely many points, called boundary vertices. We summarize the above facts:

3.2. Lemma. Let $G$ be a discrete Möbius group acting on $B^{n}$ with $V\left(B^{n} / G\right)<\infty$. Suppose that $0 \notin$ Fix $G$ and let $P$ be a normal fundamental polyhedron centered at 0 . Then $P$ is of the form

$$
P=B^{n} \backslash \bigcup_{i=1}^{2 k} \bar{B}^{n}\left(x_{i}, r_{i}\right)
$$

where each $S_{i}=S^{n-1}\left(x_{i}, r_{i}\right), i=1, \ldots, 2 k$, is orthogonal to $S^{n-1}, r_{i}=r_{i+k}$, and $T_{i} S_{i}=S_{i+k}$ for some $T_{1}, \ldots, T_{k} \in G$. Furthermore, each $T_{i}, i=1, \ldots, k$, is of the form $T_{i}=E_{i} \circ I_{i}$ where $I_{i}$ denotes the reflection in $S_{i}$ and $E_{i} \in O(n)$. 
3.3. Simple fundamental polyhedron. Let $G$ be a discrete Mobius group acting on $B^{n}$ with $V\left(B^{n} / G\right)<\infty$. A normal fundamental polyhedron $P$ for $G$ is said to be simple if no two boundary vertices of $P$ are $G$-equivalent. In other words, $P$ is simple if and only if for each boundary vertex $p \in \bar{P} \cap S^{n-1}$ all the $(n-1)$-faces of $P$ which meet at $p$ are pairwise $G$-equivalent. By [4, Lemma 3.5], $G$ has always simple fundamental polyhedra. To understand the action of $G$ near a boundary vertex $p$ of a simple fundamental polyhedron $P$ centered at $x_{0} \in B$, choose a Möbius transformation $A$ with $A B^{n}=H^{n}, A(p)=\infty$, and $A\left(x_{0}\right)=e_{n}$. Then $P_{1}=A P$ is a simple fundamental polyhedron centered at $e_{n}$ for the group $G_{1}=A G A^{-1}$ with a boundary vertex at $\infty$. The $(n-1)$-faces of $P_{1}$ which meet at $\infty$ are pairwise equivalent via elements of $G_{1}$ which generate the stabilizer $G_{\infty}=\left\{g \in G_{1}: g(\infty)=\infty\right\}$. Each $g$ in $G_{\infty} \backslash\{\mathrm{id}\}$ is a similarity in $R^{n}$ with a unique fixed point at $\infty$ and acts on each $(n-1)$-plane $\partial H^{n}(h), h>0$, in the same manner, see [4]. The normal fundamental polyhedron $P_{2}$ for $G_{\infty}$ centered at $e_{n}$ is of the form $P_{2}=Q \times(0, \infty)$ where $Q$ is a finite bounded convex euclidean $(n-1)$-dimensional polyhedron. There exists $h_{0}>0$ such that $P_{1} \cap H^{n}(h)=P_{2} \cap H^{n}(h)$ for all $h \geqq h_{0}$.

For the sake of notational simplicity we shall from now on restrict our considerations to the case $n=3$. The extension to the general case $n>3$ and $n=2$ is quite straightforward.

3.4. Lemma. Let $G$ be a discrete Möbius group acting on $B^{3}$ with $V\left(B^{3} / G\right)<\infty$. Suppose that $G$ has a simple fundamental polyhedron $P$ centered at 0 . Then there exist a finite convex euclidean 3-dimensional polyhedron $Q \subset B^{3}$ with all its vertices in $S^{2}$ and a homeomorphism $h: \bar{P} \rightarrow \bar{Q}$ such that

(i) $h \mid P$ is quasiconformal,

(ii) $h \circ T_{i}(x)=E_{i} \circ h(x)$ for all $x \in S_{i} \cap \partial P, i=1, \ldots, k$, where $S_{i}, T_{i}$, and $E_{i}$ are as in Lemma 3.2.

Proof. Case 1: $B^{3} / G$ is compact. Let $z_{1}, \ldots, z_{m}$ be the vertices of $P$ and let $\varphi_{1}$ and $\varphi_{2}$ be the radial linear stretchings defined by $P$ and the euclidean polyhedron $Q$ which is spanned by $\varphi_{1}\left(z_{1}\right), \ldots, \varphi_{1}\left(z_{m}\right)$, respectively. Then $h=\varphi_{2}^{-1} \circ \varphi_{1}$ is the required mapping. Indeed, $h$ maps $\bar{P}$ homeomorphically onto $\bar{Q}$, and since $P$ and $Q$ satisfy the $\beta$-cone condition for some $\beta>0, \varphi_{1}$ and $\varphi_{2}$ are quasiconformal by Corollary 2.8 and consequently so is $h \mid P$. For (ii) let $x \in S_{i} \cap \partial P$. Then by Lemma 3.2

$$
h \circ T_{i}(x)=h \circ E_{i} \circ I_{i}(x)=h \circ E_{i}(x) .
$$

Since $\left|x_{i}\right|=\left|x_{i+k}\right|$ and $r_{i}=r_{i+k}$, it follows by the nature of $h \mid \partial P$ and $E_{i}$, see 2.9 (c), that $h \circ E_{i}(x)=E_{i} \circ h(x)$ and so (ii) follows.

Case 2: $B^{3} / G$ is non-compact. Since now $P$ does not satisfy the $\beta$-cone condition for any $\beta>0$, we first map $P$ quasiconformally onto a domain $R \subset B^{3}$ which satisfies the $\beta$-cone condition and then proceed as in Case 1 .

Let $p_{1}, \ldots, p_{q}$ be the boundary vertices of $P$. For each $j=1, \ldots, q$ choose a Möbius transformation $A_{j}$ with $A_{j} B^{3}=H^{3}, A_{j}\left(p_{j}\right)=\infty$, and $A_{j}(0)=e_{3}$. Pick 
$h>0$ such that $A_{j}^{-1}\left(H^{3}(h)\right) \cap A_{l}^{-1}\left(H^{3}(h)\right)=\emptyset$ for $j \neq l$ and so that $\cup A_{j}^{-1}\left(H^{3}(h)\right)$ does not contain any vertex of $P$. By 3.3 each set $C_{j}=A_{j}(P) \cap H^{3}(h)$ is of the form $Q_{j} \times(h, \infty)$ where $Q_{j}$ is a convex, bounded, and finite 2-dimensional euclidean polyhedron containing 0 . Let $\psi_{j}^{*}: R^{2} \rightarrow R^{2}$ be the radial linear stretching defined by $Q_{j}$ and let $\psi_{j}: R^{2} \times[h, \infty) \rightarrow H^{3}$ be the mapping $\psi_{j}(x, s)=\left(\psi_{j}^{*}(x), s-h\right)$. Then $\psi_{j}$ is bi-lipschitzian and hence quasiconformal and maps $C_{j}$ onto the semi-infinite cylinder $Z=B^{2} \times(0, \infty)$. Next we construct a quasiconformal mapping of $Z$ onto a finite cylinder.

Let $\left(r, \varphi, x_{3}\right)$ and $(t, \varphi, \Theta)$ denote the cylinder and spherical coordinates of $R^{3}$, respectively. The polar angle is measured from the positive half of the $x_{3}$-axis. The mapping $f_{1}: Z \rightarrow B^{3} \cap H^{3}$ which is defined by $t=e^{-x_{3}}, \varphi=\varphi$, and $\Theta=\pi r / 2$ is quasiconformal, see [3, p. 47], surjective, and maps the discs $B^{2} \times\left\{x_{3}\right\}, x_{3}>0$, onto the hemispheres $S^{2}\left(e^{-x_{3}}\right) \cap H^{3}$. Let $f_{2}$ denote the reflection in the sphere $S^{2}\left(e_{2} / 3,2 / 3\right)$. Then $f_{2}$ is anti-conformal and maps $B^{3} \cap H^{3}$ onto $H^{3} \backslash \bar{B}^{3}\left(e_{2} / 2,1 / 2\right)$, $B^{3}(1 / 3) \cap H^{3}$ onto $\left\{x \in H^{3}: x_{2}<-1 / 3\right\}$ and the 2-planes through the $x_{3}$-axis onto the spheres centered on the line $L=\left\{x \in \partial H^{3}: x_{2}=-1 / 3\right\}$ and passing through the points $e_{2} / 3$ and $-e_{2}$. Using cylinder coordinates $\left(r, \varphi, x_{1}\right)$ with $L$ as the axis of symmetry and $\varphi$ measured from the direction of the vector $-e_{2}$ to the direction of $e_{3}$, we define a mapping $f_{3}: H^{3} \rightarrow H^{3}$ by

$$
\begin{aligned}
f_{3}\left(r, \varphi, x_{1}\right) & =\left(r, \pi / 2+\varphi / 4, x_{1}\right), \quad 0 \leqq \varphi<2 \pi / 3, \\
& =\left(r, \varphi, x_{1}\right), \quad 2 \pi / 3 \leqq \varphi<\pi .
\end{aligned}
$$

Then $f_{3}$ is quasiconformal, maps $H^{3} \backslash \bar{B}^{3}\left(e_{2} / 2,1 / 2\right)$ onto $\left\{x \in H^{3}: x_{2}>-1 / 3\right\}$ $\backslash \bar{B}^{3}\left(e_{2} / 2,1 / 2\right)$, maps each sphere which is centered on $L$ and which passes through the points $e_{2} / 3$ and $-e_{2}$ into itself, and maps half-planes through $L$ rigidly into half-planes through $L$.

Finally, let $\psi=f_{1}^{-1} \circ f_{2}^{-1} \circ f_{3} \circ f_{2} \circ f_{1}$. Then $\psi$ maps the semi-infinite cylinder $Z$ quasiconformally onto the finite cylinder $Z^{\prime}=B^{2} \times(0, \log 3)$ and has the following properties:

(a) $\psi$ has a homeomorphic extension to $\bar{Z}$ denoted again by $\psi$,

(b) $\psi(x)=x$ for $x \in \partial Z \cap \partial H^{3}$, and

(c) each plane through the $x_{3}$-axis is mapped into itself in the same manner.

To map $P$ onto a domain $R$ in $B^{3}$ which satisfies the $\beta$-cone condition for some $\beta>0$, define $\varphi: P \rightarrow B^{3}$ by $\varphi(x)=A_{j}^{-1} \circ \psi_{j}^{-1} \circ \psi \circ \psi_{j} \circ A_{j}(x)$ for $x \in P \cap A_{j}^{-1}\left(H^{3}(h)\right)$, $j=1, \ldots, q$, and $\varphi(x)=x$ otherwise. Then $\varphi$ is quasiconformal with a homeomorphic extension, denoted by $\varphi$, to $\bar{P}$. The image domain $R=\varphi P$ is obtained from $P$ by cutting away balls tangent to $S^{2}$ at the boundary vertices and hence $R$ satisfies the $\beta$-cone condition for some $\beta>0$. Because of (c),

$$
\varphi \circ E_{i}(x)=E_{i} \circ \varphi(x)
$$

for every $x \in \partial P \cap S_{i}$. 
Let $\left\{z_{1}, \ldots, z_{m}\right\}$ be the set of all vertices of $P$ and let $\varphi_{1}$ and $\varphi_{2}$ be the radial linear stretchings defined by $R$ and by the euclidean polyhedron $Q$ which is spanned by $\left\{\varphi_{1}\left(z_{1}\right), \ldots, \varphi_{1}\left(z_{m}\right), p_{1}, \ldots, p_{q}\right\}$, respectively. Finally, let $h=\varphi_{2}^{-1} \circ \varphi_{1} \circ \varphi$. Then $h$ maps $P$ quasiconformally onto $Q$ with a homeomorphic extension to $\bar{P}$.

To prove (ii) note that, by Lemma 3.2 and by the symmetry of $Q$, see 2.9 (c),

$$
h \circ T_{i}(x)=h \circ E_{i} \circ I_{i}(x)=h \circ E_{i}(x)=\varphi_{2}^{-1} \circ \varphi_{1} \circ E_{i}(x)=E_{i} \circ \varphi_{2}^{-1} \circ \varphi_{1}(x)=E_{i} \circ h(x)
$$

for $x \in \partial P \backslash \cup A_{j}^{-1}\left(H^{3}(h)\right)$. Suppose now that $x \in \partial P \cap A_{j}^{-1}\left(H^{3}(h)\right) \cap S_{i}$ for some $j$ and $i$. Then $h(x)=\varphi_{2}^{-1} \circ \varphi_{1} \circ \varphi(x)$ and hence by (3.5) and Lemma 3.2, $h \circ T_{i}(x)=$ $=\varphi_{2}^{-1} \circ \varphi_{1} \circ E_{i} \circ \varphi(x)$. By the same reason as above we can now interchange $E_{i}$ and $\varphi_{2}^{-1} \circ \varphi_{1}$. This yields (ii) and the proof is complete.

\section{Construction of a $Q M$ automorphic mapping in $B^{3}$}

4.1. Simplices in $R^{3}$. Given a simplex $\sigma=\left(x^{0}, x^{1}, x^{2}, x^{3}\right)$ in $R^{3}$ we let $|\sigma|$ denote the closed tetrahedron in $R^{3}$ which is spanned by the vertices $x^{0}, x^{1}, x^{2}, x^{3}$ and let $\mathbf{C}|\sigma|=\bar{R}^{3} \backslash$ int $|\sigma|$. All 3-simplices in $R^{3}$ will be oriented by the sign of the standard determinant function associated with the basis $e_{1}, e_{2}, e_{3}$.

4.2. Lemma. Given any two simplices $\sigma=\left(x^{0}, x^{1}, x^{2}, x^{3}\right)$ and $\tau=\left(y^{0}, y^{1}, y^{2}, y^{3}\right)$ in $R^{3}$ there exists a sense-preserving homeomorphism $h=h_{\sigma \tau}$ from $|\sigma|$ into $\bar{R}^{3}$ such that

(i) $h(|\sigma|)=|\tau|$ if $\sigma$ and $\tau$ have the same orientation and $h(|\sigma|)=\mathbf{C}|\tau|$ otherwise,

(ii) $h\left(x^{i}\right)=y^{i}, i=0,1,2,3$,

(iii) $h|\partial| \sigma \mid$ is a piecewise linear homeomorphism,

(iv) $h \mid$ int $|\sigma|$ is quasiconformal.

Proof. If $\sigma$ and $\tau$ have the same orientations, then the piecewise linear map of $|\sigma|$ onto $|\tau|$ which is defined by (ii) satisfies (i), (iii), and (iv).

Suppose now that $\sigma$ and, $\tau$ have different orientations. We may assume that $0 \in$ int $|\tau|$. The radial linear stretching $\varphi$ defined by int $|\tau|$ is quasiconformal in $R^{3}$ and has a quasiconformal extension to $\bar{R}^{3}$ with $\varphi(\infty)=\infty$. Let $I$. denote the reflection in $S^{2}$ and $h_{1}:|\sigma| \rightarrow|\tau|$ the sense-reversing piecewise linear map which satisfies $h_{1}\left(x^{i}\right)=y^{i}, i=0,1,2,3$. Then it is easy to check that $h=\varphi^{-1} \circ I \circ \varphi \circ h_{1}$ is the required map.

4.3. Proof of Theorem 1.2 for $n=3$. Let $P$ be a simple fundamental polyhedron for $G$ centered at $x_{0} \in B^{3}$. We may assume that $x_{0}=0$, otherwise consider first $A G A^{-1}$ for some Möbius transformation $A$ with $A B^{3}=B^{3}$ and $A\left(x_{0}\right)=0$.

Let $T_{i}$ and $E_{i}, i=1, \ldots, k$, be as in Lemma 3.2 and $Q$ and $h: \bar{P} \rightarrow \bar{Q}$ as in Lemma 3.4. Using planes through 0 triangulate $Q$ so that any two $E_{i}$-equivalent faces in $\partial Q$ have $E_{i}$-equivalent sub-triangulations. In this triangulation, call it $K$, identify $E_{i}$-equivalent faces in $\partial Q$ and thus denote vertices of $K$ which are $E_{i}$-equiv- 
alent, $i=1, \ldots, k$, by the same symbols. Now $K$ can be made fine enough so that all four vertices of any 3-simplex are distinct. Let $S=\left\{\sigma_{1}, \ldots, \sigma_{v}\right\}$ be the set of all 3 -simplices in $K$ and $\left\{0, x^{2}, \ldots, x^{N}\right\}$ the set of all vertices in $K$. Then each $\sigma_{i}$ is of the form $\left(0, x^{i_{1}}, x^{i_{2}}, x^{i_{3}}\right)$. Choose $N$ points $0, y^{2}, \ldots, y^{N}$ in general position in $R^{3}$, i.e. no four points are coplanar, and associate with each 3 -simplex $\sigma_{i}=\left(0, x^{i_{1}}, x^{i_{2}}, x^{i_{3}}\right)$ in $S$ the simplex $\tau_{i}=\left(0, y^{i_{1}}, y^{i_{2}}, y^{i_{3}}\right)$. Now define $g: \bar{Q} \rightarrow \bar{R}^{3}$ by $g|| \sigma_{i} \mid=h_{\sigma_{i} \tau_{i}}$ for $i=1, \ldots, v$. Here $h_{\sigma_{i} \tau_{i}}$ is as in Lemma 4.2.

Finally let $f: B^{3} \rightarrow \bar{R}^{3}$ be defined for $x \in T^{-1}(\bar{P}) \cap B^{3}, T \in G$, by $f(x)=g \circ h \circ T(x)$. Then $f$ is continuous by Lemma 3.4 (ii) and by the construction of $K$ and $g . f$ is automorphic with respect to $G$, and since $g \circ h$ is $q m$ in $P$ by Lemma 3.4 and Lemma 4.2, it follows that so is $f$ in $B^{3}$.

4.4. Remark. The automorphic mapping $f$ constructed above has the property $N(A, f)<\infty$ where $A$ is any fundamental set for $G$ and $N(A, f)=\sup \operatorname{card}\left(f^{-1}(y) \cap A\right)$ over all $y \in \bar{R}^{3}$.

\section{References}

[1] Alexander, J. W.: Note on Riemann spaces. - Bull. Amer. Math. Soc. 26, 1920, $370-372$.

[2] Garland, H., and M. S. Raghunathan: Fundamental domains for lattices in rank one semisimple Lie groups. - Proc. Nat. Acad. Sci. U.S.A. 62, 1969, 309-313.

[3] Gehring, F. W., and J. VÄISÄLÄ: The coefficients of quasiconformality of domains in space. Acta Math. 114, 1965, 1-70.

[4] Martio, O., and U. Srebro: Automorphic quasimeromorphic mappings in $R^{n}$. - Acta Math. $135,1975,221-247$.

[5] Selberg, A.: Recent developments in the theory of discontinuous groups of motions of symmetric spaces. - Proc. 15th Scand. Congress. Oslo 1968, Lecture Notes in Mathematics 118, Springer-Verlag, Berlin-Heidelberg-New York, 1970.

[6] Wielenberg, N.: On the fundamental polyhedra of discrete Möbius groups. - To appear.

\author{
University of Helsinki \\ Department of Mathematics \\ SF-00100 Helsinki 10 \\ Finland
}

\author{
Technion - Israel Institute \\ of Technology \\ Department of Mathematics \\ Haifa \\ Israel
}

Received 10 March 1976 